\title{
Fatores que influenciam a aprendizagem de conceitos matemáticos em cursos de engenharia: Um estudo exploratório com estudantes da Universidade do Minho
}

\author{
Manuela Alvesi, Clara Coutinhoii, Ana Maria Rochaiii \& \\ Cristina Rodriguesiv \\ Universidade do Minho, Portugal
}

\section{Resumo}

O sucesso na aprendizagem da Matemática no ensino superior constitui um campo emergente de pesquisa, em particular nos cursos de Engenharia. No entanto, em Portugal ainda é incipiente a pesquisa na área, pelo que é pertinente explorar fatores que podem esclarecer a relação entre os estudantes de Engenharia e a Matemática. Com base em três focus group realizados com estudantes de dois cursos de Engenharia da Universidade do Minho, este estudo exploratório pretende investigar as atitudes dos estudantes na aprendizagem de conceitos matemáticos e averiguar os fatores que influenciam a sua experiência no processo de aprendizagem. Para além da perceção da importância da Matemática no seu curso e na sua futura profissão, os resultados sugerem que há fatores que influenciam o seu desempenho, nomeadamente o papel do professor e a sua metodologia de ensino, bem como a aplicabilidade dos conceitos matemáticos no contexto da Engenharia, o papel ativo do estudante na aprendizagem e a influência dos pais e da sociedade.

\section{Palavras-chave}

Aprendizagem de conceitos matemáticos; Engenharia; Focus group; Ensino Superior 


\section{Introdução}

O rápido desenvolvimento tecnológico conduz a maiores níveis de competitividade, por via da maior disponibilidade de informação e da diminuição dos tempos de análise e de resposta necessários à sua gestão. Face a uma sociedade cada vez mais rápida, aumentam as exigências ao nível da educação e da investigação nas CTEM (Ciência, Tecnologia, Engenharia e Matemática) e daí a preocupação dos educadores e investigadores, que se reflete nos inúmeros estudos reportados na literatura. No ensino superior, a Matemática tem um papel de destaque nos cursos de base tecnológica. Do currículo do primeiro ano constam Unidades Curriculares - UC - da área da Matemática que são fundamentais para que os estudantes adquiram os conhecimentos-base necessários às UC mais específicas de cada curso. Sem essa base Matemática bem consolidada, o sucesso nas UC aplicadas é bastante afetado.

No caso particular da Engenharia, a Matemática desempenha um papel importante, uma vez que está ligada a diversas áreas primordiais da vida quotidiana tais como a mecânica, a construção civil, a computação, a tecnologia, a energia, os dispositivos eletrónicos e em todos os processos de fabrico. Muitos aspetos da atividade de Engenharia compreendem a formulação de problemas e a escolha de métodos adequados para resolvêlos. Independentemente da área de estudo, os conceitos matemáticos são essenciais na formação de engenheiros, quer na compreensão dos diferentes conceitos, quer no conhecimento específico da sua aplicabilidade. Durante um curso de Engenharia, os estudantes aprendem e consolidam os princípios básicos de Matemática para resolver problemas práticos, reforçando o conhecimento de conceitos matemáticos, nomeadamente das áreas de estatística, métodos numéricos, otimização e simulação, entre muitos outros.

No entanto, e apesar de a Matemática constituir uma disciplina-base na admissão a cursos de Engenharia, são identificadas dificuldades por parte dos estudantes de Engenharia nas UC com base matemática. São diversos os estudos que evidenciam as dificuldades sentidas pelos estudantes de Engenharia na transferência dos conhecimentos matemáticos para o contexto da Engenharia (ver, por exemplo: Fadali, Velasquez-Bryant, \& Robinson, 2004; Gynnild, Tyssedal, \& Lorentzen, 2005). Da experiência de muitos 
professores resulta o reconhecimento de que as notas dos seus estudantes de Engenharia revelam dificuldades e questões motivacionais que poderão ir muito além do conhecimento matemático necessário.

Em Portugal, a preocupação com o fracasso escolar tem desempenhado um papel de relevo na pesquisa em educação, tendo sido apontados diversos fatores que o explicam, como sejam a organização do currículo, a metodologia de ensino, a natureza dos conceitos ensinados, a idade e património cultural e académico da família nuclear (pais), entre outros (ver, por exemplo: Alarcão, 2000; Buescu, 2012; Domingos, 2003; Tavares et al., 2000). No entanto, a maioria dos estudos que incidem sobre o sucesso/insucesso na aprendizagem da Matemática centram-se nos fatores demográficos e envolvem sobretudo estudantes dos ensinos básico e secundário (Brites-Ferreira, Seco, Canastra, Dias, \& Abreu, 2011; Fonseca, Valente, \& Conboy, 2011; Lencastre, Guerra, Lemos, \& Pereira, 2000; Tavares \& Huet, 2001).

Embora os estudos publicados se centrem no insucesso no ensino básico e secundário, identifica-se uma preocupação gradual com esta problemática no contexto do ensino superior (Brites-Ferreira et al., 2011). Alguns estudos têm sido desenvolvidos no âmbito da problemática da educação matemática nos cursos de Engenharia. A este propósito destacamse os estudos de Bigotte de Almeida e Pessoa (2011) e Bigotte de Almeida, Fidalgo, e Rasteiro (2012), que salientam que a falta de preparação dos estudantes que ingressam em cursos de Engenharia condiciona a aprendizagem de conceitos matemáticos nos primeiros anos do curso.

Neste contexto, parece-nos pertinente identificar os fatores que afetam a aprendizagem de conceitos matemáticos no ensino superior em Portugal, em particular no contexto específico do ensino de Engenharia, uma área com uma componente matemática forte e de reconhecido impacto sobre a aplicação dos desenvolvimentos matemáticos.

Estudos recentes mostram que é importante saber a opinião dos estudantes sobre a Matemática em geral e verificar quais os fatores que os estudantes identificam como influenciadores do seu desempenho em UC com base matemática (Alves, Rodrigues, \& Rocha, 2012a; 2012b; Flegg, Mallet, \& Lupton, 2012; Harris et al., 2015). Foi este o objetivo que motivou o estudo exploratório apresentado no presente artigo, em que se procurou responder 
às seguintes questões de partida: quais as atitudes dos estudantes de Engenharia perante a aprendizagem de conceitos matemáticos? Que fatores os estudantes consideram ser influenciadores do seu desempenho na aprendizagem de conceitos matemáticos? Para o efeito, foram desenvolvidas diversas sessões de focus group com estudantes de Engenharia, uma vez que se trata de uma técnica de pesquisa qualitativa através da qual se procura obter múltiplas perspetivas acerca de uma mesma temática e em que a interação face a face funciona como elemento potenciador do emergir de novas ideias e opiniões (Greenbaum, 1998; Oliveira \& Freitas, 1998).

Com este artigo pretende-se investigar de que forma os estudantes de Engenharia percecionam a importância da Matemática, no seu curso e na sua futura profissão. Espera-se ainda contribuir para a discussão sobre os fatores que poderão influenciar a aprendizagem de conceitos matemáticos nos cursos de Engenharia.

\section{A Matemática nos cursos de Engenharia}

A Matemática tem uma multiplicidade de atributos que vão desde o desenvolvimento do raciocínio lógico de uma pessoa até à compreensão de estruturas abstratas. Ela promove o pensamento lógico e racional e aumenta a capacidade de analisar e resolver problemas. Os conceitos matemáticos surgem no currículo de muitos cursos, incluindo cursos de Engenharia, e revela-se essencial para a formação de todos os futuros engenheiros, independentemente da área de estudo e de trabalho. Dada a rápida evolução dos processos de ensino e as necessidades da Engenharia, educadores e investigadores estão constantemente a rever o currículo de Matemática nos cursos de Engenharia, com o objetivo de criar cursos com qualidade e cada vez mais adaptados às exigências da sociedade atual. Um bom exemplo disso são os esforços do Grupo de Trabalho da Matemática SEFI (SEFIMWG) para definir as necessidades matemáticas relevantes nos cursos europeus de Engenharia. O objetivo deste grupo de trabalho é assegurar que os futuros engenheiros desenvolvam um conjunto de competências, aprendam e consolidem os princípios básicos da Matemática que os ajudarão a solucionar os diversos problemas práticos que Ihes surgirão na sua futura profissão (Alpers, 2013; Fuller, 2002; Mustoe, 2002). 
André (2008) considera que a Matemática desempenha um tríplice papel na formação superior de um engenheiro: como escola de pensamento, como linguagem e como ferramenta. Como escola de pensamento, a Matemática ajuda o estudante a aprender a pensar e a comunicar o seu pensamento aos outros com objetividade, rigor e concisão. A Matemática é para um estudante de Engenharia a linguagem natural, e não só está presente na Física (a sua ciência experimental básica) como em todas as ciências da Engenharia. Como ferramenta, a Matemática compreende diversas técnicas analíticas e numéricas para resolver eficazmente os problemas matemáticos que resultam da "escrita em linguagem matemática" de problemas de Engenharia (André, 2008). A ligação entre as UC da formação de base matemática e as UC da área da matemática aplicada é de extrema importância, na medida em que uma boa formação matemática de base tem uma influência benéfica na formação específica de Engenharia ao longo do curso. A correlação entre estes dois grupos de UC deve ser forte e positiva para que ao estudante seja conferida uma verdadeira formação matemática aplicada à Engenharia (Yassin \& Amin, 2009).

\section{Fatores que influenciam a aprendizagem de conceitos matemáticos no ensino superior}

Diversos estudos evidenciam que, no ensino superior, em particular nos cursos de Engenharia, a maior percentagem de retenção se verifica em UC baseadas na aquisição de conceitos matemáticos. Este fenómeno tem sido objeto de estudo de várias investigações (Alarcão, 2000; Bakar et al., 2010; Bogaard, 2011; Domingos, 2003; Parsons \& Adams, 2005, Tavares et al., 2000). Este facto pode estar relacionado com o próprio insucesso da disciplina de Matemática nos ensinos básico e secundário. Há autores que defendem que os conceitos matemáticos aprendidos no ensino secundário têm uma índole mais operacional ou prática, pelo que a capacidade para a aprendizagem de conceitos matemáticos mais abstratos, lecionados no ensino superior, é reduzida e, segundo Domingos (2003), "manifesta-se sobretudo na manipulação de objectos matemáticos definidos simbolicamente (...) sendo a compreensão dos mesmos feita de forma parcial" (p. 1). O mesmo autor defende que o desempenho dos estudantes vai diminuindo à medida que os conceitos se vão tornando mais abstratos. Neves e Teodoro (2013) referem mesmo que 
(...) apesar de indissociavelmente relacionadas com os fenómenos do mundo real, a base primordial dos modelos mentais humanos, as estruturas de conhecimento e cognição da CTEM envolvem quadros conceptuais e metodológicos abstratos que desafiam o senso comum do conhecimento de todos os dias e tornam difíceis os correspondentes processos de ensino e aprendizagem. (p. 37)

Existem assim evidências de que a formação matemática no ensino secundário poderá não ir ao encontro das exigências do ensino superior. Mesmo os melhores estudantes revelam dificuldades ao nível da compreensão dos conceitos e da resolução de problemas. Em muitos casos, em disciplinas da área da Engenharia, a falha na resolução de problemas advém da incapacidade dos estudantes em realizar manipulações algébricas simples (Mustoe, 2002, 2006). No entanto, para Alarcão (2000),

(...) a atribuição de culpas ao ensino básico e secundário ou aos estudantes, se bem que tenha algum fundamento, é explicação demasiado simplista para poder sequer explicar o fenómeno e, muito menos, contribuir para a sua resolução. Igualmente simplista, mas que por vezes com algum fundamento, é a atribuição de culpas à falta de competência pedagógica dos docentes do ensino superior, afirmação que hoje, infelizmente, ameaça transformar-se num chavão. (p. 14)

A motivação dos estudantes para a aprendizagem de conceitos matemáticos é uma das principais preocupações dos educadores e dos investigadores em educação. A motivação é a força motriz que está por detrás das nossas ações e que condiciona as nossas necessidades, desejos e ambições na vida. Estudos mostram existir uma relação entre a motivação para a obtenção de resultados e o desempenho académico de estudantes, no ensino superior (Bakar et al., 2010; Sirmaci, 2010; Walter \& Hart, 2009). Por outro lado, os estudos ao nível da aprendizagem de conceitos matemáticos nos cursos de Engenharia indiciam que a mesma pode ser condicionada por vários fatores, nomeadamente o género (Meelissen \& Luyten, 2008; Tariq, Qualter, Roberts, Appleby, \& Barnes, 2013), a perceção da Matemática (Suthar, Tarmizi, Midi, \& Adam, 2010; Winkelman, 2009), o professor, a sua metodologia de ensino e o currículo das disciplinas (Flegg et al., 2012; Ramli, Shafie, \& Tarmizi, 2013), a opinião de pais/sociedade/pares (Muir, 2012), bem como as atitudes e crenças (Bakar et al., 2010; Kargar, Tarmizi, \& Bayat, 2010; Sirmaci, 2010). 


\subsection{Género}

Desde os anos 70, séc. XX, tem vindo a ser investigada a questão do género e a sua influência na performance académica dos estudantes e na atitude perante a Matemática (Brites-Ferreira et al., 2011; Patterson et al., 2003). É ideia geral que os estudantes do sexo masculino são melhores na Matemática do que os estudantes do sexo feminino (Feingold, 1988; Halpern, 1997). Outros estudos mostram que a competência para a Matemática é igual em ambos os géneros nos primeiros anos de ensino, sendo que, em anos mais avançados, os estudantes do sexo feminino demonstram mais interesse pela Matemática e melhores resultados (Fouad \& Smith, 1996). As diferenças entre os géneros na aptidão para a Matemática podem ser explicadas por vários fatores, nomeadamente a ideia pré-concebida, que é imbuída desde cedo nas crianças, de que os estudantes do sexo masculino são melhores a Matemática do que os estudantes do sexo feminino (Meelissen \& Luyten, 2008). No entanto, o estereótipo tradicional que favorece os estudantes do sexo masculino na Matemática tem mudado ao longo dos anos. Estudantes do sexo masculino tendem a superar os do sexo feminino nos testes padronizados de performance matemática, especialmente em populações do ensino secundário e universitário; no entanto, as mulheres frequentemente superam os seus colegas do sexo masculino quando se comparam as notas em sala de aula, evidenciando que a diferença entre os sexos está a diminuir (Arnup, Murrihy, Roodenburg, \& McLean, 2013).

Estudos sobre o género e a performance matemática mostram que o estereótipo tradicional que favorece os homens em detrimento das mulheres na proficiência matemática diminuiu acentuadamente ao longo dos últimos 40 anos e as diferenças de género no desempenho matemático já não são uma questão relevante (Brandell \& Staberg, 2008; Meelissen \& Luyten, 2008; Plante, Théorêt, \& Favreau, 2009). De acordo com Brandell e Staberg (2008), Meelissen e Luyten (2008) e Plante, Théorêt, e Favreau (2009), investigações sobre o género e a Matemática são frequentemente limitadas à relação entre as diferenças de género e as atitudes perante a Matemática e entre as diferenças de género e a performance matemática. Embora haja estudos que demonstram não existir uma diferença significativa entre os sexos no que concerne à motivação matemática e às atitudes em relação à Matemática, é possível verificar em muitos outros que o género está relacionado com a 
performance matemática (Moenikia \& Zahed-Babelan, 2010). Patterson et al. (2003) referem também que o género por si só pode não explicar diferenças significativas no desempenho, quando visto no contexto de vários tipos de conhecimento matemático, havendo uma crescente necessidade de explorar outros fatores que poderão estar na origem das dificuldades sentidas na aprendizagem de conceitos matemáticos.

\subsection{Perceção da importância da Matemática}

A motivação dos estudantes para a aprendizagem de conceitos matemáticos é influenciada pela perceção que os estudantes têm sobre a Matemática em geral e, no caso específico da Engenharia, a sua aplicabilidade no contexto da sua futura profissão (Flegg et al., 2012; Goold \& Devitt, 2012).

Winkelman (2009), num estudo acerca das perceções sobre a Matemática em Engenharia, verificou que, apesar de a maioria dos estudantes verem a Matemática como uma porta de entrada para o curso de Engenharia, para muitos trata-se de uma disciplina difícil, destinada apenas a estudantes com talento, o que impede muitos deles de entrarem para cursos de Engenharia.

Craig (2013) refere que os estudantes do primeiro ano de Engenharia não compreendem o papel que a Matemática irá ter no seu curso e na sua futura profissão. Os estudantes entram para a universidade com conceções simplistas e ingénuas sobre a Matemática, havendo, para o autor, uma necessidade de desenvolver conceções mais maduras em sala de aula. A perceção que os estudantes têm sobre a natureza e o papel da Matemática no seu curso e na sua futura profissão é pedagogicamente importante e tem um impacto na aprendizagem, sendo uma potencial influência na forma de ensinar e nos conteúdos que são ensinados (Wood, 2008; Wood et al., 2012). Wood (2008) defende que, uma vez que a noção de futuras aplicações influencia a forma como o estudante se envolve e aplica, é importante que os professores expandam proativamente a perceção dos estudantes sobre a importância da Matemática na sua futura carreira como engenheiros. Em relação às perceções dos estudantes acerca da importância da Matemática na sua futura carreira profissional, o investigador salienta que alguns dos 
estudantes reconhecem que é importante durante o seu curso adquirir competências, tais como a resolução de problemas e o raciocínio lógico, para se tornar num bom profissional, enquanto para outros estudantes essa ligação não é clara. Assim, é necessário, por parte do professor, um maior esforço em estabelecer a ligação entre os conceitos matemáticos adquiridos pelos estudantes e a sua futura carreira profissional (Wood, 2008).

\subsection{Metodologia/professor/currículo}

A metodologia de ensino utilizada pelos professores é um dos fatores que surgem na literatura como tendo influência na forma como os estudantes aprendem (Sauer \& Soares, 2004; Swan, 2005; Valdez, 2012). Relativamente ao ensino superior, Valdez (2012) refere que os educadores têm implementado vários instrumentos para envolver melhor os estudantes, incluindo aprendizagem ativa e colaborativa, comunidades de aprendizagem, aprendizagem de serviço, educação cooperativa, inquérito e aprendizagem baseada em problemas e projetos de equipa.

Existe uma grande diversidade na forma como os estudantes aprendem, quer seja a observar e a ouvir, a refletir e a agir, através de raciocínios lógicos ou intuitivos ou através da memorização. Tendo em conta esta diversidade, os métodos de ensino devem ser igualmente variados. Há professores que demonstram ou discutem; alguns evidenciam regras e outros destacam exemplos; alguns enfatizam a memória e outros a compreensão. Aquilo que um determinado estudante aprende numa aula é ajustado, em parte, pela abordagem e pelos pré-requisitos, mas também pela compatibilidade do método de aprendizagem e caraterísticas do professor na abordagem ao ensino. Podem ocorrer algumas incompatibilidades profundas entre os estilos de aprendizagem dos estudantes numa aula e o estilo de ensino do professor. Essas incompatibilidades podem levar os estudantes a ficar aborrecidos e desatentos na aula e a ter mau desempenho nos testes, ficando desanimados com o curso e podendo concluir que não são bons e acabar por desistir. Os professores, confrontados com o baixo desempenho, com turmas que não respondem ou são hostis ou desistentes, podem tornarse excessivamente críticos com os seus estudantes ou começar a questionar a sua própria competência como docentes (Valdez, 2012). 
Flegg et al. (2012) referem no seu estudo que vários investigadores centram a sua atenção na forma como a Matemática deve ser ensinada nos cursos de Engenharia. Há diferentes opiniões acerca do tipo de abordagem e de ensino dos conceitos matemáticos. Alguns autores defendem que a ênfase do ensino deve ser no seu aspeto formal e rigoroso (Kent \& Noss, 2003), enquanto outros refutam essa ideia (Noskov \& Shershneva, 2007). A forma como os estudantes são expostos aos conceitos matemáticos desempenha um papel fulcral no seu desempenho. Se a conexão entre os conceitos matemáticos e a sua aplicabilidade no contexto da sua área profissional não for evidente, os estudantes tendem a perder o interesse pela disciplina (Firouzian, Ismail, Rahman, \& Yusof, 2012). O uso de estratégias de resolução de problemas adaptados ao contexto real é necessário para que os estudantes se interessem pelas disciplinas de base matemática (Dall'Alba, 2009; Flegg et al., 2012).

Harris et al. (2015) defendem a ideia de que é importante que os próprios engenheiros sejam parte ativa no processo de definição das disciplinas a serem lecionadas nos cursos de Engenharia, para que se fomente a aplicação dos conceitos matemáticos abstratos no contexto prático da Engenharia. Para os autores, o ensino da Matemática deve estar sempre ligado ao seu "valor de uso" para a Engenharia, o que se consegue com o recurso a exemplos que a contextualizem, tornando-a mais interessante para os estudantes (Harris et al., 2015, p. 334).

Sauer e Soares (2004) referem que, nos cursos de Engenharia, a apresentação dos conceitos matemáticos é feita sob a forma de regras e fórmulas, execução de algoritmos, informações sobre definições, teoremas e linguagem simbólica. Uma das consequências dessa forma de apresentação dos conceitos matemáticos é a passividade, a insegurança do estudante e a sua incapacidade de decidir, sem a ajuda do professor, se os resultados obtidos estão ou não corretos. Neste cenário, aprender significa assistir a aulas, observar o que é feito pelo professor, copiar e repetir procedimentos. De entre as capacidades e competências que um engenheiro deve desenvolver destacam-se o raciocínio lógico, saber ler e interpretar esquemas, gráficos e a linguagem matemática, sintetizar informações e desenvolver processos na resolução de problemas, bem como expressar-se de forma clara e organizada. Ensinar apenas por meios expositivos e teóricos 
não é suficiente para que estas aptidões e competências sejam desenvolvidas. O trabalho docente não consiste apenas em transmitir informações ou conhecimentos, mas em apresentá-los sob a forma de problemas a resolver, contextualizando-os e colocando-os em perspetiva, de modo a que o estudante consiga estabelecer a ligação entre a sua solução e outras perguntas mais abrangentes (Valdez, 2012).

\subsection{Influência dos pais e da sociedade}

As crenças e a opinião dos pais e da sociedade em geral sobre a Matemática aparecem referenciadas na literatura como fatores que influenciam a perceção dos estudantes sobre a Matemática e, consequentemente, sobre o seu desempenho (Cai, 2003; Civil, 2001; Cobb, 1986; Muir, 2011, 2012; Walkerdine, 1990).

A perceção dos pais e da sociedade em geral sobre a Matemática influencia igualmente a motivação dos estudantes e a sua atitude perante esta disciplina. Muitos pais tendem a valorizar as suas próprias formas de fazer matemática sobre a Matemática escolar, enquanto as crianças valorizam os conhecimentos aprendidos na escola em detrimento dos que obtêm dos pais, podendo isto levar ao surgimento de potenciais tensões aquando da resolução de tarefas matemáticas em casa (Muir, 2011, 2012). Atitudes negativas da sociedade em relação à Matemática e dificuldade percebida do seu estudo levam os estudantes a escolher cada vez menos a Matemática a favor de outras disciplinas (Gordon \& Nicholas, 2012).

Pritchard (2004) sintetizou uma série de estudos que concluíram que havia ligações entre as atitudes, perceções e crenças dos pais sobre a Matemática e as atitudes e desempenho dos estudantes em Matemática. As atitudes dos pais influenciam o desempenho dos estudantes na Matemática e os pais transmitem as suas crenças e atitudes sobre a Matemática através das suas práticas individuais (Pritchard, 2004). Resultados de outras pesquisas indicam que muitos adultos, em relação a tarefas matemáticas, admitem sentimentos de ansiedade, impotência, medo e aversão (ver, por exemplo, Haylock, 2007). Isto é preocupante, na medida em que muitas vezes são os próprios pais que passam estes sentimentos aos seus filhos. Embora muitos desses pais considerem a Matemática importante, também tendem a 
desvalorizá-la por incidir demasiado na memorização de regras e procedimentos (ver, por exemplo, Onslow, 1992).

\subsection{Crenças e atitudes}

Bruinsma e Jansen (2007) referem que as crenças dos estudantes são um importante contributo para a obtenção de resultados positivos. Os estudantes que acreditam que podem fazer a UC tendem a ter notas mais altas. Assim, crenças mais robustas conduzem o indivíduo a uma maior motivação durante a realização de tarefas (Bandura et al., 2008; Walter \& Hart, 2009; Zimmerman, Bandura, \& Martinez-Pons, 1992). Segundo Bandura et al. (2008), as crenças têm uma influência na ação, na motivação e nos processos cognitivos, sendo estes últimos relacionados com a antecipação de consequências e resultados de ações. No contexto escolar, essas crenças podem afetar a motivação dos estudantes para realizar as tarefas ou evitá-las, as reações que esses apresentam diante das suas realizações e até mesmo as suas escolhas profissionais. Um dos aspetos importantes, e sobre o qual já existem alguns estudos, é determinar se existe uma relação entre as crenças matemáticas e os resultados obtidos por estudantes do ensino superior. Há várias evidências que mostram que as crenças que os estudantes têm sobre a Matemática são preponderantes no desenvolvimento de carreiras ligadas à Matemática. No entanto, ainda existe pouca literatura acerca deste fenómeno no contexto do ensino superior (Gordon \& Nicholas, 2012; Suthar et al., 2010).

Hodges e Kim (2013) definem atitude perante a Matemática como uma medida que avalia a reação à Matemática, em determinados contextos. Os autores argumentam que os fatores motivacionais, como o interesse e o valor da tarefa, constituem-se como atitudes. A atitude em relação à Matemática pode interferir futuramente na autoestima, na formação de identidade e na relação com a utilidade dessa disciplina na profissão (Hodges \& Kim, 2013). Estas investigações concluíram que o nível de desempenho do estudante pode ser relacionado com a atitude positiva do mesmo em relação à Matemática e, mesmo que o estudante com uma atitude positiva não apresente um alto nível de desempenho, este será melhor do que o obtido pelo estudante que mostrou uma atitude negativa. A partir do momento em que as atitudes do estudante em relação a um conteúdo são favoráveis, ele 
poderá estar altamente motivado para aprender. Além disso, pode investir em esforços mais intensos e mais concentrados durante o processo de ensinoaprendizagem. Mas, quando as atitudes são desfavoráveis, é possível que esses fatores venham a ter um efeito contrário.

\section{Metodologia e amostra}

O estudo aqui apresentado é de natureza exploratória. O seu principal objetivo é aferir a opinião dos estudantes sobre a Matemática em geral e sobre os fatores que, na sua perspetiva, são influenciadores do seu desempenho em UC com base matemática.

O focus group é uma técnica de pesquisa qualitativa, através da qual se procura obter uma série de perspetivas acerca de uma mesma temática. Assim, escolhemos esta técnica por ser a mais adequada à recolha dos dados (Greenbaum, 1998; Oliveira \& Freitas, 1998). Uma vez que fatores como as crenças e as atitudes, a motivação, a influência de pais e sociedade, a metodologia de ensino, o professor e o currículo das disciplinas surgem referenciados na literatura como influenciadores da aprendizagem, elaborámos o guião de questões à luz desses fatores, bem como de resultados obtidos em outros estudos (Alves et al., 2012a, 2012b).

Os focus group foram realizados na Universidade do Minho, tendo-se usado amostras de conveniência oriundas de dois cursos de Engenharia desta universidade. A escolha dos cursos foi intencional. Segundo Greenbaum (1998) e Oliveira e Freitas (1998), a escolha dos participantes no estudo deve ser cuidada e feita de acordo com o propósito da pesquisa.

O primeiro focus group envolveu estudantes do $3^{\circ}, 4^{\circ}$ e $5^{\circ}$ anos do Mestrado Integrado em Engenharia e Gestão Industrial (MIEGI), num total de 10 estudantes - 5 do género feminino e 5 do género masculino -, com idades compreendidas entre os 21 e os 24 anos. Dada a especificidade do curso de MIEGI (com uma forte componente de gestão e, por isso, com uma componente matemática mais aplicada), foi do nosso interesse reproduzir o focus group com outros estudantes de Engenharia de cursos com maior base tecnológica e com maior necessidade de aplicação de conceitos matemáticos. Assim, um segundo e um terceiro focus group foram realizados, cada um com 14 estudantes do $2^{\circ}$ ano do curso de Mestrado Integrado em Engenharia 
Mecânica (MIEMEC), o que resultou num total de 28 estudantes - 24 do género masculino e 4 do género feminino -, com idades entre os 19 e os 32 anos. A diversidade do grupo espelha as dificuldades dos estudantes, uma vez que estas duas entrevistas de grupo foram realizadas no final de uma aula de Estatística Aplicada com estudantes que ficaram a tirar dúvidas com o docente. Na literatura, o tamanho do grupo de discussão é importante, uma vez que devemos ter em conta que seja pequeno o suficiente para que todos tenham a oportunidade de partilhar as suas opiniões e grande o bastante para fornecer uma diversidade de opiniões (Oliveira \& Freitas, 1998).

Cada focus group iniciou-se com a apresentação do tema do estudo e da equipa de investigadores, a identificação dos objetivos do estudo, a garantia da confidencialidade e o consentimento informado. Seguiu-se a entrevista de grupo, realizada em duas fases: 1) uma discussão orientada por um guião com perguntas abertas; 2) o comentário a três afirmações apresentadas pela investigadora.

As questões apresentadas aos estudantes (ver Quadro 1) tiveram o objetivo de iniciar a discussão do tema de forma a estes exprimirem as suas opiniões acerca da importância da Matemática nos cursos de Engenharia, as suas atitudes perante a aprendizagem dos conceitos matemáticos e as suas perceções acerca do próprio desempenho nas disciplinas de base matemática.

\section{Quadro 1: Guião de questões do focus group}

\begin{tabular}{|l|l|}
\hline \multicolumn{1}{|c|}{ Objetivos } & \multicolumn{1}{c|}{ Questões } \\
\hline $\begin{array}{l}\text { Confirmação } \\
\text { Contextualização } \\
\text { Experiência prévia }\end{array}$ & $\begin{array}{l}\text { (i) No geral, gostam de Matemática? Porquê? } \\
\text { (ii) E nos anos de escolaridade anteriores à entrada na } \\
\text { universidade? Que tipo de alunos foram a Matemática e } \\
\text { às disciplinas de ciências em geral? }\end{array}$ \\
\hline Atitudes & $\begin{array}{r}\text { (iii) A Matemática "pesou" (bem ou mal) no momento de } \\
\text { escolher o curso? }\end{array}$ \\
\hline Normas subjetivas & $\begin{array}{r}\text { Acham que a Matemática é importante no vosso futuro } \\
\text { profissional? }\end{array}$ \\
\hline Normas individuais intrínsecas & $\begin{array}{l}\text { Na vossa opinião, que fatores interferem na } \\
\text { aprendizagem dos conceitos matemáticos? Aluno - } \\
\text { professor - currículo - instituição? } \\
\text { (vi) Na vossa opinião, a opinião dos pais e da sociedade em } \\
\text { geral interfere na opinião dos alunos sobre a Matemática? }\end{array}$ \\
\hline $\begin{array}{l}\text { (vii) Perante a necessidade de aprendizagem de conceitos } \\
\text { matemáticos, o que vos motiva/desmotiva? }\end{array}$ \\
\hline
\end{tabular}


As questões (i) e (ii) (Quadro 1) serviram o propósito de contextualizar os estudantes na temática que ia ser discutida, por um lado, e, por outro, são questões confirmatórias, uma vez que já era do nosso conhecimento que estes estudantes gostavam de Matemática, que eram bons estudantes a Matemática, razão pela qual escolheram um curso de Engenharia.

As questões (iii) e (iv) (Quadro 1) visaram aferir as atitudes dos estudantes perante a Matemática no que concerne à importância que a mesma teve na sua decisão de optar por um curso de Engenharia. Segundo Ajzen e Fishbein (1980), a "atitude em relação a um conceito é simplesmente o grau de favorabilidade ou não favorabilidade a este conceito" (p. 54).

As questões (v) e (vi) (Quadro 1) dizem respeito às normas subjetivas. Segundo Ajzen e Fishbein (1980), as normas subjetivas dizem respeito à "perceção do sujeito quanto à pressão social exercida sobre a mesma para que realize ou não realize um determinado comportamento" (p. 6). A norma subjetiva é determinada pelas crenças normativas do indivíduo, isto é, a perceção do que os outros referentes pensam acerca do que o indivíduo deve ou não deve fazer, e, por outro lado, pela motivação que o indivíduo tem para corresponder às expectativas desses referentes (Ajzen, 1985).

A questão (vii) (Quadro 1) teve como objetivo aferir as crenças individuais de cada estudante sobre a aprendizagem dos conceitos matemáticos.

De salientar ainda que o guião inicial serviu apenas de base orientadora da discussão. As respostas às questões tomaram rumos diferentes nos três focus group, uma vez que, à medida que a discussão se desenrolava, outros temas foram surgindo.

Numa segunda fase do focus group, tal como referido anteriormente, apresentaram-se três afirmações para comentar, cujo objetivo foi o de "provocar" os estudantes no sentido de conseguir uma discussão mais aberta do tema e na qual pudessem surgir mais opiniões sobre a problemática. As afirmações foram as seguintes:

(i) A Matemática é vista como um obstáculo e um instrumento de seleção em vários cursos superiores.

(ii) Os piores resultados não são em tarefas de cálculo, são nas tarefas mais complexas, que exigem mais raciocínio, flexibilidade e espírito crítico. 
(iii) Os estudantes do sexo masculino são melhores a Matemática do que os do sexo feminino, logo são melhores engenheiros!

As sessões de focus group foram gravadas e transcritas pelas investigadoras com base em técnicas de análise de conteúdo. Para proceder à categorização optámos por procedimentos dinâmicos que combinaram a leitura do corpus e a literatura. Assim sendo, na nossa investigação, de natureza exploratória, as categorias foram sendo descobertas a par do desenvolvimento do percurso de contextualização teórica e de investigação, tal como sugerido por Carmo e Ferreira (1998). Este revelar-se-ia um processo dinâmico, criativo e envolvente, por parte das investigadoras, que contemplou a criação de uma grelha de análise e a introdução/associação das respetivas unidades de análise. A unidade de análise escolhida foi o "tema", tal como definido por Bardin (2008) e Esteves (2006). As unidades de análise são secções de texto de natureza e dimensão variáveis que devem afigurarse como elementos detentores de um sentido completo e com pertinência para o objeto de estudo. Podem apresentar-se sob forma de unidade de registo, que se define como "a unidade de significação a codificar ao segmento de conteúdo a considerar como unidade base, visando a categorização e a contagem frequencial" (Bardin, 2008, p. 130).

Segundo Carmo e Ferreira (1998, p. 257), dentro das unidades de registo podemos considerar as "unidades formais", de nível linguístico (a palavra, a frase, uma personagem, etc.) e a "unidade semântica" (o tema). No âmbito da análise de conteúdo, ambas podem fornecer dados relevantes; porém, a utilização do tema ganha maior pertinência se considerarmos que "é a unidade de significação que se liberta naturalmente de um texto analisado segundo certos critérios relativos à teoria que serve de guia à leitura", mas também porque habitualmente é usado "para estudar motivações de opiniões, de atitudes, de valores, de crenças, de tendências, etc.", sendo aplicado a diversos instrumentos de recolha de dados, nomeadamente em entrevistas não diretivas ou mais estruturadas (Bardin, 2008, p. 131).

Cada depoimento foi tomado como uma unidade de contexto que informou o posterior recorte com base na unidade de análise escolhida: 0 tema (Bardin, 2008). Assim, resolvemos atribuir um código a cada depoimento que permitisse identificar a qual dos cursos pertencia o inquirido (A - MIEGI; $B$ - MIEMEC). Como as categorias emergiram de diferentes fontes de 
evidência - opiniões resultantes da discussão orientada pelas questões e comentários a afirmações -, resolvemos ainda atribuir a cada opinião dada o código $Q$ e a cada comentário às afirmações o código F. A partir das unidades de registo que foram codificadas em cada categoria, foi possível extrair um número variável de indicadores que ajudam a compreender melhor o sentido de cada categoria. Os indicadores apresentam as inferências do investigador a partir das unidades de registo apresentadas. $O$ processo de categorização incluiu uma fase de verificação e melhoria da fidelidade da categorização, o que foi conseguido através da verificação e confirmação por dois investigadores independentes e conhecedores do estudo em curso que analisaram a grelha de categorias e subcategorias criada. A posterior discussão entre os investigadores envolvidos no estudo levou a um reajuste final da grelha inicial (Coutinho, 2013).

Da análise feita emergiram então duas categorias. A primeira categoria, que designámos por $A$ Matemática e a Engenharia, inclui os indicadores relativos à importância da Matemática na escolha do curso e à importância da Matemática na futura profissão. No que concerne à segunda categoria, Fatores influenciadores da aprendizagem de conceitos matemáticos, evidenciaram-se: o professor/metodologia/tipologia de aula; a natureza dos conceitos matemáticos; a aplicabilidade das matérias; a opinião dos pais/sociedade/pares; e o género.

\section{Resultados}

Conforme referido anteriormente, a implementação dos focus group visou obter informação para as duas questões fundamentais do nosso estudo: i) quais as atitudes dos estudantes de Engenharia perante a aprendizagem de conceitos matemáticos; e ii) que fatores influenciam o seu desempenho nas UC de base matemática.

A análise é feita por categoria e ilustrada com pequenos extratos das verbalizações dos estudantes no decurso das sessões dos focus group. Recorda-se que a cada depoimento foi atribuído um código que permitisse identificar a qual dos cursos pertencia o inquirido ( $\mathrm{A}$ - MIEGI; B - MIEMEC). Como as categorias emergiram de diferentes fontes de evidência - opiniões resultantes da discussão orientada pelas questões e comentários a 
afirmações -, foi igualmente atribuída a cada opinião o código $Q$ e a cada comentário às afirmações o código $\mathrm{F}$.

\subsection{A Matemática e a Engenharia}

A primeira categoria evidencia, em geral, a relevância da Matemática nos cursos de Engenharia. A maioria dos estudantes que participaram nos focus group é da opinião que a Matemática é fundamental nos cursos de Engenharia, independentemente da área de estudo. Relativamente à importância que a Matemática teve na escolha do curso, os estudantes consideram-na uma disciplina essencial na escolha do curso. Alguns estudantes mencionaram:

(...) se não tivéssemos Matemática, escolher um curso de Engenharia estaria fora de questão. (BQ)

Se não gostasse de Matemática provavelmente não teria escolhido o curso de MIEGI. (AQ)

No que concerne à importância da Matemática na futura profissão, os estudantes referiram que se trata de uma área do saber fundamental ao percurso académico e profissional. Os estudantes têm consciência de que a aprendizagem de conceitos matemáticos é fundamental para atingir o sucesso, não só nas UC de Matemática, mas também nas outras UC onde os conceitos matemáticos são fundamentais para resolver problemas mais diretamente ligados à sua Engenharia. Foi referido, por exemplo:

[A Matemática] é necessária para resolver problemas reais e é o fundamento de muitos sistemas industriais. (BF)

(...) a Matemática é importante para o nosso futuro e, considerando que o nosso curso tem uma parte de gestão, convém sabermos Matemática. (AQ)

\subsection{Fatores que influenciam a aprendizagem de conceitos matemáticos}

Relativamente aos fatores que os participantes no estudo consideram ser influenciadores da aprendizagem de conceitos matemáticos, reconhecem que o método de ensino adotado pelo professor, o currículo das disciplinas e a tipologia da aula têm um efeito direto na sua motivação e consequente 
desempenho. Os participantes reconhecem que o professor é um fator que interfere na motivação para a aprendizagem dos conceitos matemáticos, tendo inclusive alguns dos participantes admitido que por vezes não se sentem apoiados por alguns professores: ou pela falta de feedback, ou pelo método de ensino baseado na memorização de conceitos. Apresentamos algumas evidências:

O método do professor é importante e pode interferir na nossa maneira de encarar a disciplina e até o nosso desempenho. (BQ)

Há professores que têm o cuidado de, ao fazer os exercícios, os aplicar a situações reais e que no futuro poderemos usar... assim os exercícios ficam mais interessantes. (BQ)

Os estudantes participantes nos focus group referiram a falta de aplicabilidade das matérias como uma causa para a sua falta de motivação e interesse e consequente mau desempenho. Os estudantes concordaram que o curriculum das UC é importante e que deve ser mais direcionado para as necessidades profissionais dos estudantes, i.e., ao nível dos estudos aplicados em Engenharia. Quando isso não acontece, os estudantes sentemse mais dispersos e desmotivados. Foi mencionado que:

Grande parte da Matemática lecionada em determinados cursos nada tem a ver com o curso propriamente dito. (BF)

(...) temos cadeiras [UC] em que fazemos exercícios que nós sabemos perfeitamente que nunca mais vamos usar e que são só para "encher"... que são só para termos matéria para fazer teste. (BQ)

Em algumas cadeiras [UC] abordamos assuntos que não têm aplicação prática na nossa futura profissão. (AQ)

A natureza dos conceitos matemáticos é igualmente influenciadora da motivação e do desempenho. Os estudantes admitem ainda ter dificuldades na compreensão dos conceitos matemáticos nas aulas teóricas pela falta de contextualização prática, expressando o desejo de as UC com base matemática abordarem conceitos com mais aplicabilidade na sua futura prática profissional. As aulas teóricas, nos seus moldes expositivos, requerem, pois, um maior cuidado ao nível da motivação e perceção da sua importância. Na sua generalidade, os estudantes são da opinião de que as grandes dificuldades na Matemática são a interpretação e o raciocínio crítico. 
Nos anos que antecedem o ensino superior não se privilegia este tipo de problemas, que envolvam mais raciocínio e espírito crítico, e quando os estudantes se deparam pela primeira vez com UC onde essa componente matemática mais abstrata existe, os seus resultados académicos descem consideravelmente. A este respeito os estudantes mencionaram:

A Matemática torna-se exigente quando deixa de ser apenas cálculo e começa a envolver raciocínio, pois deixa de ser metódica e mecânica para ser mais abstrata e ambígua. (BF)

Nos anos do secundário somos habituados a fazer exercícios sempre do mesmo género e depois tudo o que vai além disto... para a maioria dos alunos torna-se difícil resolver problemas. (BF)

A influência dos pais e da sociedade em geral é reconhecida como um elemento que pode interferir no desempenho na Matemática, pela desculpabilização dos maus resultados. Os participantes admitem que a sociedade é em parte responsável pela opinião negativa que se tem da Matemática e que, no caso particular da opinião dos pais, este pode ser um fator bastante importante e significativo na opinião dos filhos sobre a disciplina:

Eu acho que em geral toda a gente odeia Matemática. (...) se os pais não percebem, e se as crianças vão para casa com dificuldades, os pais não vão poder ajudar. (BQ)

A maioria dos estudantes concorda que a sociedade em geral vê a Matemática como um obstáculo, o que leva a que muitos estudantes condicionem as suas escolhas quando se trata de optarem por cursos com uma forte componente matemática:

(...) nos dias de hoje muitos alunos trazem já esta noção de dificuldade da Matemática e muitas vezes isso exerce peso na escolha do curso superior. (AF)

A opinião dos pares é igualmente importante. Os estudantes reconhecem que se houver uma ideia preconcebida sobre a dificuldade de uma UC isso irá interferir na forma como encaram a disciplina e o seu empenho:

A opinião dos nossos colegas às vezes interfere na nossa visão sobre uma cadeira... Se nos disserem que é um cadeirão, nós já vamos com medo. (BQ) 
[A opinião dos colegas] pode servir de motivação porque nos obriga a estudar mais, mas podemos ficar desmotivados porque temos medo de não conseguirmos... (BQ)

Relativamente ao género, na perspetiva dos participantes, este não parece ser fator determinante nem na aprendizagem de conceitos matemáticos nem no sucesso na sua futura profissão. No entanto, a maioria dos estudantes do sexo masculino considera que as estudantes (do sexo feminino) são mais organizadas e metódicas no que concerne ao estudo, o que pode levar a que obtenham melhores resultados, ou seja, trata-se de uma questão de método de estudo e não de género. A este respeito, um estudante afirmou:

(...) não se trata de uma questão de género mas apenas uma questão pessoal, que tudo tem a ver com o método de estudo de cada um. Talvez as mulheres sejam mais organizadas... (BF)

O mesmo estudante acrescentou, contudo, que, para se ser bom engenheiro, não basta ter métodos de trabalho e ser bom estudante a Matemática, havendo outros fatores que podem influenciar o processo:

(...) embora as raparigas tenham tendência a ser mais aplicadas e estudiosas, o que vai dar origem a um bom engenheiro não é apenas ser bom na Matemática, mas depende de outros fatores. (BF)

Já as estudantes, embora reconhecendo que prevalece na sociedade, em geral, a ideia de que os rapazes são melhores estudantes a Matemática e que isso determina a opção por cursos de Engenharia, consideram tratar-se de um preconceito que corresponde cada vez menos à realidade nos cursos de Engenharia:

(...) por não haver tantas raparigas a escolher Engenharia, como os rapazes... Principalmente se for Engenharia Informática, ou Civil, ou Mecânica... Mas no nosso curso isso até não acontece. (AF)

(...) esse preconceito deve-se às percentagens de mulheres nos cursos de Engenharia, o que nada tem a ver com a realidade. Na verdade, em termos de notas, as raparigas têm-se mostrado melhores alunas do que os rapazes. (BF)

Torna-se assim evidente que, na opinião dos estudantes participantes no estudo, o estereótipo tradicional que favorece os estudantes do sexo masculino como melhores no desempenho em Matemática e, logo, mais 
propensos a ter sucesso em cursos de forte base matemática, como é o caso da Engenharia, não é uma realidade.

\section{Discussão dos resultados}

Baseado nas opiniões de estudantes de cursos de Engenharia da Universidade do Minho e na literatura que aborda esta temática, este estudo pretende contribuir para a discussão da problemática da aprendizagem de conceitos matemáticos em cursos de Engenharia, em Portugal. Esta é uma área de estudo pouco abordada nas investigações portuguesas e que, a nível internacional, constitui uma temática atual e relevante para a educação em Engenharia. Assim sendo, e tomando como base a revisão de literatura sobre fatores que influenciam a aprendizagem de conceitos matemáticos, passamos a discutir os resultados obtidos nos focus group realizados a estudantes de Engenharia da Universidade do Minho.

O primeiro dado a assinalar é que, de uma forma mais ou menos explícita, todos os fatores a que alude a literatura emergem das palavras dos estudantes. Começando pela questão do género, os resultados do nosso estudo, em linha com os estudos de Arnup et al. (2013) e Meelissen e Luyten (2008), sugerem a mudança no estereótipo tradicional que favorece os estudantes do sexo masculino como melhores no desempenho em Matemática e, logo, mais propensos a ter sucesso em cursos de forte base matemática, como é o caso da Engenharia. Contudo, os dados sugerem diferenças associadas ao género nas justificações apontadas pelos estudantes, verificando-se que os rapazes, embora reconhecendo os melhores resultados em Matemática obtidos pelas raparigas, consideram que isso se deve não a questões de género mas a outros fatores, como sejam os hábitos de trabalho e métodos de estudo por parte das estudantes. Consideram também que o facto de elas terem melhores notas a Matemática não significa que venham a ser melhores engenheiras. As estudantes, por seu turno, consideram que o preconceito que favorece a performance dos estudantes do sexo masculino no desempenho na Matemática, e que não corresponde à realidade dos factos - as raparigas que cursam Engenharia na Universidade do Minho têm melhores notas a Matemática do que os estudantes do sexo masculino -, continua a determinar as escolhas do curso e explica o reduzido número de estudantes do sexo feminino que escolhem 
Engenharia como opção no ensino superior. Em suma, em consonância com o verificado por Patterson et al. (2003), os participantes no estudo consideram que o género, por si só, poderá não explicar diferenças no desempenho, havendo que investigar outros fatores que as justifiquem.

Relativamente à segunda dimensão em análise, os resultados mostram que os estudantes de Engenharia que participaram no estudo reconhecem a importância da Matemática, quer na escolha do curso, quer no decorrer da sua futura profissão. Para muitos destes estudantes, tal como reportado por Winkelman (2009), a Matemática é uma porta de entrada para o curso de Engenharia, influenciando de forma determinante a escolha desse curso. No que diz respeito às perceções sobre a importância da Matemática na futura profissão, os estudantes consideram tratar-se de uma área do saber fundamental, tanto no percurso académico como no profissional. Os estudantes têm consciência de que a aprendizagem de conceitos matemáticos é fundamental para atingir o sucesso, não só nas UC de Matemática, mas também nas outras UC onde os conceitos matemáticos são fundamentais para resolver problemas mais diretamente ligados à Engenharia. Trata-se de dados encorajadores para o caso da Engenharia, uma vez que, na perspetiva de diversos autores (Flegg et al., 2012; Goold \& Devitt, 2012), a opinião que os estudantes têm sobre a Matemática em geral e o reconhecimento da sua importância e da sua aplicabilidade ao contexto da futura profissão são determinantes na aprendizagem de conceitos matemáticos.

Verifica-se, assim, que a perceção que os estudantes têm sobre a natureza e o papel da Matemática no seu curso e na sua futura profissão tem implicações pedagógicas (Wood, 2008; Wood et al., 2012). Nesse sentido, no nosso estudo, os participantes reconhecem que o professor é um fator que poderá interferir na motivação para a aprendizagem dos conceitos matemáticos, tendo inclusive alguns dos estudantes admitido que, por vezes, não se sentem apoiados, seja pela falta de feedback, seja pelo método de ensino, muito baseado na memorização de conceitos e sem revelar a aplicabilidade a contextos reais de Engenharia. Um dos resultados mais interessantes deste estudo é, aliás, o facto de os estudantes admitirem que se sentem tanto mais motivados quanto mais prática é a aplicação dos conceitos matemáticos abordados nas UC de base matemática. Estes resultados estão 
em linha com os resultados obtidos nos estudos congéneres de Harris et al. (2015) que defendem um ensino integrado da Matemática com a Engenharia. Harris et al. (2015) acreditam que, com este tipo de abordagem, se aumenta a disposição dos estudantes para a aprendizagem da Matemática, levando a menores taxas de retenção no primeiro ano do curso.

Neste sentido, emerge do estudo que a tipologia de aula e o método de ensino constituem, no juízo dos participantes, fatores que influenciam a sua aprendizagem de conceitos matemáticos e desempenho académico. Existem, igualmente, evidências na literatura que mostram que o método de ensino usado com estudantes de Engenharia afeta a forma como estes encaram a própria Matemática. Temos o exemplo do estudo de Swan (2005), no qual o autor distingue dois tipos de pedagogia: a "transmissionista" (do inglês, transmissionist) e a "conexionista" (do inglês, connectionist). A primeira refere-se à transmissão simples dos conhecimentos através de exemplos, onde as explicações dos professores são prevalentes - o ensino centra-se principalmente nos resultados processuais. A segunda abordagem centra-se numa pedagogia mais colaborativa que recorre ao uso de tarefas desafiadoras que enfatizam a natureza interligada da Matemática, muitas vezes envolvendo o debate - o ensino centra-se mais na compreensão dos conceitos; esta abordagem baseia-se no conhecimento prévio dos estudantes e permite-lhes, mais facilmente, encontrar soluções desafiadoras e nãorotineiras para problemas práticos, o que, segundo o autor supracitado, é o desejável em Engenharia.

$\mathrm{Na}$ sequência do anteriormente explanado, Flegg et al. (2012) sugerem o uso de tarefas baseadas em problemas desenvolvidos, quer por matemáticos, quer por engenheiros, num trabalho colaborativo para a conceção de um programa de Engenharia. Estes investigadores acreditam que isso potenciará, nos estudantes, o reconhecimento da relevância da Matemática nos seus estudos e futuras carreiras.

Neste estudo, e em linha com os resultados verificados por Winkelman (2009), os estudantes foram claros ao reconhecer que as maiores dificuldades na aprendizagem de conceitos matemáticos são a interpretação e raciocínio lógico, o que nos remete para a importância do papel do estudante no processo de aprendizagem. Também Boaler (2000) é de opinião que os estudantes devem aprender a fazer a conexão entre o que é aprendido nas 
UC de base matemática e a sua aplicação numa tarefa ou contexto de Engenharia. Para Putnam e Borko (2000), a forma como o sujeito aprende um conjunto particular de conceitos e desenvolve determinadas capacidades e a situação real de aplicabilidade tornam-se parte fundamental do que é aprendido. Assim, perante uma nova situação, a Engenharia requer uma resolução de problemas e modelação, e não apenas a aplicação direta dos diferentes conceitos matemáticos (Williams \& Goos, 2013). Faz pois sentido, tal como sugere Valdez (2012), que o trabalho docente em Engenharia não deva consistir apenas em transmitir informações ou conhecimentos, mas em apresentá-los sob a forma de problemas a resolver, contextualizando-os e colocando-os em perspetiva, de modo a que o estudante consiga estabelecer a ligação entre a sua solução e outras perguntas mais abrangentes.

Outro fator importante na aprendizagem de conceitos matemáticos que emergiu nas entrevistas com os estudantes é a influência dos pais e da sociedade em geral. Na opinião dos estudantes, as atitudes negativas dos pais e da sociedade em relação à Matemática levam a que cada vez menos estudantes escolham cursos com uma forte componente matemática. Este facto é evidenciado noutras investigações, por exemplo de Gordon e Nicholas (2012). Também Cobb (1986) e Walkerdine (1990) referiam que muitos dos fatores que influenciam a transferência dos conhecimentos matemáticos têm origem social. Assim, o presente estudo confirma que a crença social de que a Matemática é inacessível, complexa e de difícil compreensão poderá estar na base de uma menor dedicação à disciplina e posterior aptidão, ainda que reconhecendo que a Matemática é uma pedra basilar dos cursos de Engenharia.

\section{Conclusão}

Em Portugal, a preocupação com o fracasso escolar tem desempenhado um papel de relevo na pesquisa em educação, embora a maioria dos estudos publicados sobre o insucesso na aprendizagem de conceitos matemáticos se centrem, sobretudo, nos ensinos básico e secundário. Apesar de já existirem alguns estudos no âmbito da problemática da educação matemática nos cursos de Engenharia, de que são exemplos os de Bigotte de Almeida et al. (2012) e Bigotte de Almeida e Pessoa (2011), verifica-se, nessas abordagens, uma lacuna na investigação sobre o 
insucesso na aprendizagem de conceitos matemáticos no ensino superior, na medida em que não incluem fatores psicográficos na tentativa de compreender/explicar tal insucesso. Assim, e com a intenção de colmatar essa lacuna, consideramos oportuno abordar esses fatores psicográficos, a saber: as atitudes e crenças dos estudantes ou as suas normas subjetivas, no contexto da Engenharia - uma área com forte componente matemática e de reconhecido impacto na aplicação dos desenvolvimentos matemáticos.

No meio académico de investigação desta problemática, é reconhecida a importância da recolha da opinião dos estudantes sobre a Matemática em geral e de verificar quais os fatores que eles identificam como influenciando o seu desempenho em UC com base matemática. Foi nesse contexto que se procedeu à realização de focus group com estudantes da Universidade do Minho a frequentar cursos de Engenharia, mais especificamente os cursos de Mestrado Integrado em Engenharia e Gestão Industrial e de Mestrado Integrado em Engenharia Mecânica. Este estudo, como vimos, explorou, por um lado, as atitudes dos estudantes de Engenharia perante a aprendizagem de conceitos matemáticos e, por outro, os fatores que influenciam a aprendizagem de conceitos matemáticos e a sua aplicabilidade pelos futuros profissionais de Engenharia.

Relativamente ao fator género, a opinião dos estudantes sugere que não influencia o desempenho na sua futura profissão como engenheiros. Embora haja um reconhecimento, por parte dos estudantes, de que as raparigas podem atingir melhores resultados, esse facto poder-se-á dever a outros fatores, tais como os hábitos de trabalho e os métodos de estudo. Estes resultados vêm no seguimento dos da literatura, que identificam uma mudança no estereótipo tradicional que favorece os estudantes do sexo masculino como tendo melhor desempenho em Matemática e maior propensão ao sucesso em cursos de forte base matemática, como é o caso da Engenharia.

Analisando as opiniões dos estudantes participantes no estudo, foi ainda possível verificar que a Matemática é percecionada como sendo de extrema relevância para os cursos de Engenharia e para o desenvolvimento da sua futura profissão. Os estudantes demostraram ter consciência de que a aprendizagem de conceitos matemáticos é fundamental para atingir o sucesso, não só nas UC de Matemática, mas também nas outras UC onde os 
conceitos matemáticos são fundamentais para resolver problemas mais diretamente ligados à Engenharia.

$\mathrm{Na}$ opinião dos estudantes que participaram no estudo, poderão existir outros fatores que influenciam o seu desempenho, sendo eles o professor e o método de ensino, a tipologia de aula, a natureza dos conceitos e a aplicabilidade das matérias. É particularmente interessante concluir que os estudantes se mostram tanto mais motivados quanto mais prática é a aplicação dos conceitos matemáticos abordados nas UC de base matemática. A motivação dos estudantes para a aprendizagem de conceitos matemáticos é influenciada pela perceção que têm sobre a Matemática em geral e, no caso específico da Engenharia, sobre a sua aplicabilidade no contexto da sua futura profissão. A natureza abstrata e a não aplicabilidade dos conceitos matemáticos poderá contribuir para o insucesso nos cursos de Engenharia. Se por um lado é importante a adoção de estratégias de ensino que favoreçam uma pedagogia colaborativa, centrada no estudante e na elaboração de tarefas desafiadoras e não-rotineiras, por outro lado, é de extrema importância que os estudantes de Engenharia reconheçam que são parte integrante e ativa da aprendizagem.

A influência dos pais e da sociedade surge como outro fator importante na aprendizagem de conceitos matemáticos. Na opinião dos estudantes, as atitudes negativas dos pais e da sociedade em relação à Matemática levam a que cada vez menos estudantes escolham cursos com uma forte componente matemática, como é o caso da Engenharia.

O estudo apresentado é apenas um estudo inicial. Os resultados permitem a identificação de variáveis a serem utilizadas num futuro estudo, mais aprofundado, sobre os fatores que influenciam o sucesso dos estudantes de Engenharia em Unidades Curriculares de base matemática.

\section{Agradecimentos}

Este trabalho foi apoiado pela FCT (Fundação para a Ciência e Tecnologia, Portugal) no âmbito do projeto PEst-OE/EEI/UI0319/2014. 


\section{Referências}

Ajzen, I. (1985). From intentions to actions: A theory of planned behavior. In J. Kuhl \& J. Beckmann (Orgs.), Action control: From cognition to behavior (pp. 11-39). Berlim: Springer-Verlag.

Ajzen, I., \& Fishbein, M. (1980). Understanding attitudes and predicting social behavior. Englewood Cliffs, NJ: Prentice Hall.

Alarcão, I. (2000). Para uma conceptualização dos fenómenos de insucesso/sucesso escolares no ensino superior. In J. Tavares \& R. Santiago (Eds.), Ensino superior: (In)sucesso académico (pp. 13-23). Porto: Porto Editora.

Alpers, B. (2013). The SEFI Mathematics Working Group's new curriculum framework document. In Proceedings of the 41st SEFI Conference, 16-20 September 2013. Leuven: SEFI aisbl.

Alves, M. C., Rodrigues, C. S., \& Rocha, A. M. (2012a). Engineering students and mathematics achievement: A Portuguese case study. In S. I. Ao, L. Gelman, D. W. L. Hukins, A. Hunter \& A. M. Korsunsky (Eds.) Proceedings of the 2012 International Conference of Applied and Engineering Mathematics (ICAEM) under the World Conference on Engineering (WCE 2012), 4-6 July 2012 (pp. 8994). London: Newswood Limited.

Alves, M. C., Rodrigues, C. S., \& Rocha, A. M. (2012b). Mathematics achievement in engineering: An exploratory study with MIEGI students. In R. M. Lima, D. Carvalho, V. Cavenaghi, M. V. Junior, G. L. R. Vaccaro, R. F. M. Marçal, F. S. Másculo, \& L. F. R. R. S. Carmo (Eds.), Proceedings of XVIII International Conference on Industrial Engineering and Operations Management (ICIEOM 2012). Bauru, SP: ABEPRO.

André, J. (2008). Ensinar e estudar matemática em engenharia. Coimbra: Imprensa da Universidade de Coimbra.

Arnup, J., Murrihy, C., Roodenburg, J., \& McLean, L. (2013). Cognitive style and gender differences in children's mathematics achievement. Educational Studies, 39(3), 355-368.

Bakar, K. A., Tarmazia, R. A., Mahyuddina, R., Eliasa, H., Luana, W. L., \& Ayub, A. F. M. (2010). Relationships between university students' achievement motivation, attitude and academic performance in Malaysia. Procedia - Social and Behavioral Sciences, 2(2), 4906-4910.

Bandura, A., Caprara, G. V., Fida, R., Vecchione, M., Del Bove, G., Vecchio, G. M., \& Barbaranelli, C. (2008). Longitudinal analysis of the role of perceived selfefficacy for self-regulated learning in academic continuance and achievement. Journal of Educational Psychology, 100(3), 525-534.

Bardin, L. (2008). Análise de conteúdo ( $4^{\mathrm{a}}$ ed.). Lisboa: Edições 70.

Bigotte de Almeida, M. E., \& Pessoa, M. T. (2011). Aprender matemática nas licenciaturas de engenharia: Um estudo de caso. In A. B. Lozano, M. P. Uzquiano, A. P. Rioboo, J. C. Blanco, B. D. Silva, \& L. S. Almeida (Eds.), Atas do XI Congreso Internacional Galego-Portugués de Psicopedagoxía (pp. 34953503). Corunha: Universidade da Corunha (CD-ROM).

Bigotte de Almeida, M. E., Fidalgo, C., \& Rasteiro, D. M. (2012). Compreender o insucesso da matemática nas licenciaturas de engenharia: Um estudo de caso. 
In T. Estrela et al. (Eds.), Atas do XIX Colóquio AFIRSE - Revisitar os Estudos Curriculares: Onde estamos e para onde vamos?. Lisboa: Educação/Secção Portuguesa de AFIRSE.

Boaler, J. (2000). Exploring situated insights into research and learning. $J$ Res Math Educ., 39, 113-119.

Bogaard, M. (2011). Explaining student success in engineering education in Delft University of Technology; A synthesis of literature. In J. Bernardino \& J. C. Quadrado (Eds.), Actas do World Engineering Education 2011 (WEE2011) (pp. 236-244). Lisboa, Portugal.

Brandell, G., \& Staberg, E. M. (2008). Mathematics: A female, male or gender-neutral domain? A study of attitudes among students at secondary level. Gender and Education, 20(5), 495-509.

Brites-Ferreira, J., Seco, G. M., Canastra, F., Dias, I., \& Abreu, M. O. (2011). (In)sucesso académico no ensino superior: Conceitos, factores e estratégias de intervenção. Revista Ibero Americana de Educación Superior, 2(4), 28-40.

Bruinsma, M., \& Jansen, E. P. (2007). Educational productivity in higher education: An examination of part of the Walberg educational productivity model. School Effectiveness and School Improvement, 18(1), 45-65.

Buescu, J. (2012). Matemática em Portugal: Uma questão de educação. Lisboa: Fundação Francisco Manuel dos Santos.

Cai, J. (2003). Investigating parental roles in students' learning of mathematics from a cross-national perspective. Mathematics Education Research Journal, 15(2), 87-106.

Carmo, H., \& Ferreira, M. (1998). Metodologia de investigação: Guia para a autoaprendizagem. Lisboa: Universidade Aberta.

Civil, M. (2001). Redefining parental involvement: Parents as learners of mathematics. Paper presented at the NCTM research pre-session, Orlando, FL.

Cobb, P. (1986). Contexts, goals, beliefs and learning mathematics. Learn Math., 6, 210.

Coutinho, C. P. (2013). Metodologia de investigação em ciências sociais e humanas: Teoria e prática. Coimbra: Almedina.

Craig, T. S. (2013). Conceptions of mathematics and student identity: Implications for engineering education. International Journal of Mathematical Education in Science and Technology, 44(7), 1020-1029.

Dall'Alba, G. (2009). Investigating preparation for the professional practice. Learn. Professional, 4, 37-52.

Domingos, A. M. D. (2003). Compreensão de conceitos matemáticos avançados - A matemática no início do superior (Tese de doutoramento). Universidade Nova de Lisboa, Lisboa.

Esteves, M. (2006). A análise de conteúdo. In J. A. Lima \& J. A. Pacheco (Orgs.), Fazer investigação - Contributos para a elaboração de dissertações e teses (pp. 105126). Porto: Porto Editora. 
Fadali, M., Velasquez-Bryant, N., \& Robinson, M. (2004, October). Work in progress Is attitude toward mathematics a major obstacle to engineering education? Paper presented at The ASEE/IEEE Frontiers in Education Conference - 34th Annual Meeting, Savannah, GA.

Feingold, A. (1988). Cognitive gender differences are disappearing. American Psychologist, 43(2), 95-103.

Firouzian, S., Ismail, Z., Rahman, R. A., \& Yusof, Y. M. (2012). Mathematical learning of engineering undergraduates. Procedia - Social and Behavioral Sciences, 56, 537-545.

Flegg, J., Mallet, D., \& Lupton, M. (2012). Students' perceptions of the relevance of mathematics in engineering. International Journal of Mathematical Education in Science and Technology, 43(6), 717-732.

Fonseca, J., Valente, M. O., \& Conboy, J. (2011). Student characteristics and PISA science performance: Portugal in cross-national comparison. Procedia - Social and Behavioral Sciences, 12, 322-329.

Fouad, N. A., \& Smith, P. L. (1996). A test of social cognitive model for middle school students: Math and science. Journal of Counseling Psychology, 43(3), 338-346.

Fuller, M. (2002). The role of mathematics learning centres in engineering education. European Journal of Engineering Education, 27(3), 241-247.

Goold, E., \& Devitt, F. (2012). The role of mathematics in engineering practice and in the formation of engineers. In A. Avdelas (Ed.), Proceedings of 40th Annual Conference SEFI - Engineering Education 2020: Meet the future (pp. 252-253). SEFI: Brussels.

Gordon, S., \& Nicholas, J. (2012). Students' conceptions of mathematics bridging courses. Journal of Further and Higher Education, 37(1), 109-125.

Greenbaum, T. L. (1998). The handbook for focus group research (2nd ed.). Thousand Oaks: Sage.

Gynnild, V., Tyssedal, J., \& Lorentzen, L. (2005). Approaches to study and the quality of learning: Some empirical evidence from engineering education. Int $J$ Math Educ Sci Technol, 3, 587-607.

Halpern, D. F. (1997). Sex differences in intelligence - Implications for education. American Psychologist, 52(10), 1091-1102.

Harris, D., Black, L., Hernandez-Martinez, P., Pepin, B., Williams, J., \& The TransMaths Team (2015). Mathematics and its value for engineering students: What are the implications for teaching? International Journal of Mathematical Education in Science and Technology, 46(3), 321-336.

Haylock, D. (2007). Mathematics explained for primary teachers (3rd ed.). Thousand Oaks, CA: Sage Publications.

Hodges, C. B., \& Kim, C. (2013). Improving college students' attitudes toward mathematics. TechTrends, 57(4), 59-66.

Kargar, M., Tarmizi, R. A., \& Bayat, S. (2010). Relationship between mathematical thinking, mathematics anxiety and mathematics attitudes among university students. Procedia - Social and Behavioral Sciences, 8, 537-542. 
Kent, P., \& Noss, R. (2003). Mathematics in the university education of engineers: $A$ report to the Ove Arup Foundation (Tech. Rep.). London: Ove Arup Foundation.

Lencastre, L., Guerra, M. P., Lemos, M. S., \& Pereira, D. C. (2000). Adaptação dos alunos do primeiro ano das licenciaturas da Faculdade de Ciências da Universidade do Porto. In J. Tavares \& R. Santiago (Ed.), Ensino superior: (In)sucesso académico (pp. 75-106). Porto: Porto Editora.

Meelissen, M., \& Luyten, H. (2008). The Dutch gender gap in mathematics: Small for achievement, substantial for beliefs and attitudes. Studies in Educational Evaluation, 34(2), 82-93.

Moenikia, M., \& Zahed-Babelan, A. (2010). A study of simple and multiple relations between mathematics attitude, academic motivation and intelligence quotient with mathematics achievement. Procedia - Social and Behavioral Sciences, 2, 1537-1542.

Muir, T. (2011). Join the club: Engaging parents in mathematics education. In J. Clark, B. Kissane, J. Mousley, T. Spencer, \& S. Thornton (Eds.), Mathematics: Traditions and [new] practices - Proceedings of the 2011 AAMT-MERGA Conference (pp. 531-539). Alice Springs, NT: MERGA.

Muir, T. (2012). Numeracy at home: Involving parents in mathematics education. International Journal for Mathematics Teaching and Learning, January 25th, 1-13.

Mustoe, L. (2002). Mathematics in engineering education. European Journal of Engineering Education, 27(3), 237-240.

Mustoe, L. (2006). The mathematics background of undergraduate engineers. International Journal of Electrical Engineering Education, 39(3), 192-200.

Neves, R. G., \& Teodoro, V. D. (2013). Modelação computacional, ambientes interactivos e o ensino da ciência, tecnologia, engenharia e matemática. Revista Lusófona de Educação, 25, 35-58.

Noskov, M. V., \& Shershneva, V. A. (2007). The mathematics education of an engineer: Traditions and innovations. Russian Educ. Soc., 49, 70-84.

Oliveira, M., \& Freitas, H. M. R. (1998). Focus group - Pesquisa qualitativa: Resgatando a teoria, instrumentalizando o seu planejamento. Revista de Administração, 33(3), 83-91.

Onslow, B. (1992). Improving the attitude of students and parents through family involvement in mathematics. Mathematics Education Research Journal, 4(3), 24-31.

Parsons, S., \& Adams, H. (2005). Success in engineering mathematics... Through maths support and changes to engineering maths lectures at Harper Adams. MSOR Connections, 5(1), 1-4.

Patterson, M., Perry, E., Decker, C., Eckert, R., Klaus, S., Wendling, L., \& Papanastasiou, E. (2003). Factors associated with high school mathematics performance in the United States. Studies in Educational Evaluation, 29, 91-108.

Plante, I., Théorêt, M., \& Favreau, O. E. (2009). Student gender stereotypes: Contrasting the perceived maleness and femaleness of mathematics and language. Educational Psychology: An International Journal of Experimental Educational Psychology, 29(4), 385-405. 
Pritchard, R. (2004). Investigating parental attitudes and beliefs in mathematics education. In I. Putt, R. Faragher, \& M. McLean (Eds.), Mathematics education for the third millenium: Towards 2010 - Proceedings of the 27th Annual Conference of the Mathematics Education Research Group of Australasia (pp. 478-485). Sydney: MERGA.

Putnam R., \& Borko, H. (2000). What do new views of knowledge and thinking have to say about research on teacher learning? Educ Res., 29, 4-15.

Ramli, F., Shafie, N., \& Tarmizi, R. A. (2013). Exploring student's in-depth learning difficulties in mathematics through teacher's perspective. Procedia - Social and Behavioral Sciences, 97, 339-345.

Sauer, L. Z., \& Soares, E. M. (2004). Um novo olhar sobre a aprendizagem de matemática para a engenharia. In H. N. Cury (Org.), Disciplinas matemáticas em cursos superiores: Reflexões, relatos, propostas (pp. 245-270). Porto Alegre: EDIPUCRS.

Sirmaci, N. (2010). The relationship between the attitudes towards mathematics and learning styles. Procedia - Social and Behavioral Sciences, 9, 644-648.

Suthar, V., Tarmizi, R. A., Midi, C., \& Adam, M. B. (2010) Students' beliefs on mathematics and achievement of university students: Logistics regression analysis. Procedia - Social and Behavioral Sciences, 8, 525-531.

Swan, M. (2005). Improving learning in mathematics: Challenges and strategies. Sheffield: Teaching and Learning Division, Department for Education and Skills Standards Unit.

Tariq, V. N., Qualter, P., Roberts, S., Appleby, Y., \& Barnes, L. (2013). Mathematical literacy in undergraduates: Role of gender, emotional intelligence and emotional self-efficacy. International Journal of Mathematical Education in Science and Technology, 44(8), 1143-1159.

Tavares, J., \& Huet, I. (2001). Sucesso académico no ensino superior: Um olhar sobre o professor universitário. In R. B. Sousa, E. Sousa, F. Lemos, \& C. Januário, Pedagogia na universidade (pp. 149-160). Lisboa: Universidade Técnica de Lisboa.

Tavares, J., Santiago, R., Pinho, L. V., Pereira, A. S., Oliveira, J. A. B., Ferraz, M. F. S. \& Pereira, D. C. (2000). Factores de sucesso/insucesso no $1^{\circ}$ ano dos cursos de licenciatura em ciências e engenharias do ensino superior. In A. P. Soares, A. Osório, J. V. Capela, L. S. Almeida, R. M. Vasconcelos, \& S. M. Caires (Eds.), Transição para o ensino superior (pp. 189-214). Braga: Universidade do Minho.

Valdez, M. M. (2012). Novas metodologias no ensino e aprendizagem na área da engenharia eletrotécnica (Tese de doutoramento). Universidade do Porto, Porto.

Walkerdine V. (1990). Difference, cognition and mathematics education. Learn Math., 10, 51-56.

Walter, J. G., \& Hart, J. (2009). Understanding the complexities of student motivations in mathematics learning. The Journal of Mathematical Behavior, 2-3(28), 162-170.

Williams J., \& Goos. M. (2013). Modelling with mathematics and technologies. In K. Clements, A. Bishop, C. Keitel, J. Kilpatrick, \& F. Leung (Eds.), Third international handbook of mathematics education (pp. 549-571). London: Springer. 
Winkelman, P. (2009). Perceptions of mathematics in engineering. European Journal of Engineering Education, 34(4), 305-316.

Wood, L. N, Mather, G., Petocz, P., Reid, A., Engelbrecht, J., Harding, A., ...Perrett, G. (2012). University students' views of the role of mathematics in their future. Int $J$ Sci Math Ed., 10, 99-119.

Wood, L. N. (2008). Engineering mathematics - What do students think? ANZIAM J., 49, C513-C525.

Yassin, A. Y. M., \& Amin, N. S. (2009). The importance of engineering mathematics. In Engineering Mathematics Research Group, UKM, Bangi (Orgs.), EMaS'O9 Proceedings of Engineering Mathematics Seminar 2009: Theory and applications in engineering mathematics. Bangi: Universiti Kebangsaan Malaysia.

Zimmerman, B. J., Bandura, A., \& Martinez-Pons, M. (1992). Self-motivation for academic attainment: The role of self-efficacy beliefs and personal goal setting. American Educational Research Journal, 29(3), 663-676. 
INFLUENCING FACTORS IN THE LEARNING OF MATHEMATICAL CONCEPTS IN ENGINEERING COURSES: AN EXPLORATORY STUDY WITH STUDENTS OF THE UNIVERSITY OF MINHO

\section{Abstract}

Success in the learning of Mathematics in higher education constitutes an emerging field of research, particularly in the Engineering courses. In Portugal, however, the research in this area remains incipient. It is therefore relevant to explore which factors may clarify the relation between the Engineering students and Mathematics. Based on three focus groups carried out with students from two Engineering courses at the University of Minho, this exploratory study aims to investigate the attitudes of students in the learning of mathematical concepts and find out the factors that influence their experience in the learning process. Beyond the perception of the importance of Mathematics in their course and their future profession, the results suggest that there are factors that influence their performance, such as the teacher's role and his/her teaching methodology, as well as the applicability of mathematical concepts in the context of Engineering, the student's active role in learning and the influence of parents and society.

Keywords

Learning of mathematical concepts; Engineering; Focus group; Higher education

\section{LES FACTEURS QUI INFLUENCENT L'APPRENTISSAGE DES CONCEPTS MATHÉMATIQUES DANS LES COURS D'INGÉNIERIE: UNE ÉTUDE EXPLORATOIRE AVEC DES ÉTUDIANTS DE L'UNIVERSITÉ DE MINHO}

Résumé

Le succès de l'apprentissage de la Mathématique à l'enseignement supérieur est un domaine émergent de la recherche, particulièrement aux cours 
d'Ingénierie. Toutefois, au Portugal, la recherche est encore naissante, donc il est pertinent d'explorer les facteurs qui peuvent expliquer la relation entre les étudiants en Ingénierie et les Mathématiques. Basé sur trois groupes de discussion menés avec des étudiants de deux cours d'Ingénierie de I'Université de Minho, cette étude exploratoire vise à étudier les attitudes des élèves dans l'apprentissage des concepts mathématiques et de déterminer les facteurs qui influent sur leur expérience dans le processus d'apprentissage. En plus de la perception de l'importance des Mathématiques dans leur cours et leur future profession, les résultats suggèrent qu'il existe des facteurs qui influencent leur performance, à savoir : le rôle de l'enseignant dans la méthodologie de l'enseignement et l'applicabilité des concepts mathématiciens dans le contexte de I'Ingénierie, le rôle active de l'étudiant dans l'apprentissage et l'influence des parents et de la société.

Mots-clé

L'apprentissage des concepts mathématiques ; Ingénierie ; Groupe de discussion ; L'enseignement supérieur

Recebido em abril/2015

Aceite para publicação em novembro/2015

i Centro Algoritmi, Escola de Engenharia, Universidade do Minho, Portugal.

ii Professora Aposentada, Instituto de Educação, Universidade do Minho, Portugal.

iii Centro Algoritmi, Escola de Engenharia, Universidade do Minho, Portugal.

iv Centro Algoritmi, Escola de Engenharia, Universidade do Minho, Portugal.

Toda a correspondência relativa a este artigo deve ser enviada para: Ana Maria A.C. Rocha, Centro Algoritmi, Escola de Engenharia, Universidade do Minho, Campus de Gualtar, 4710-057 Braga, Portugal- E-mail: arocha@dps.uminho.pt 